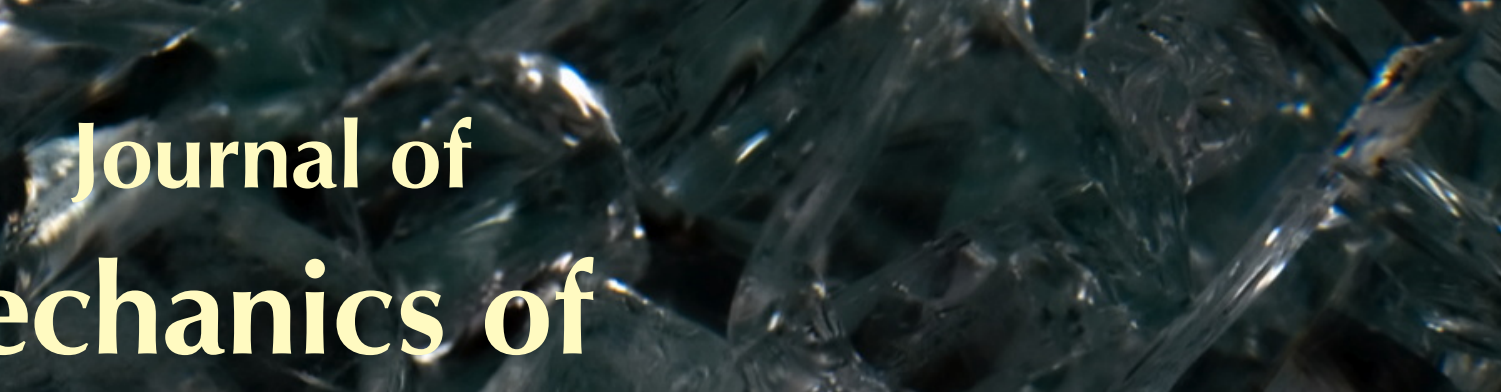

Materials and Structures

Mechanics of
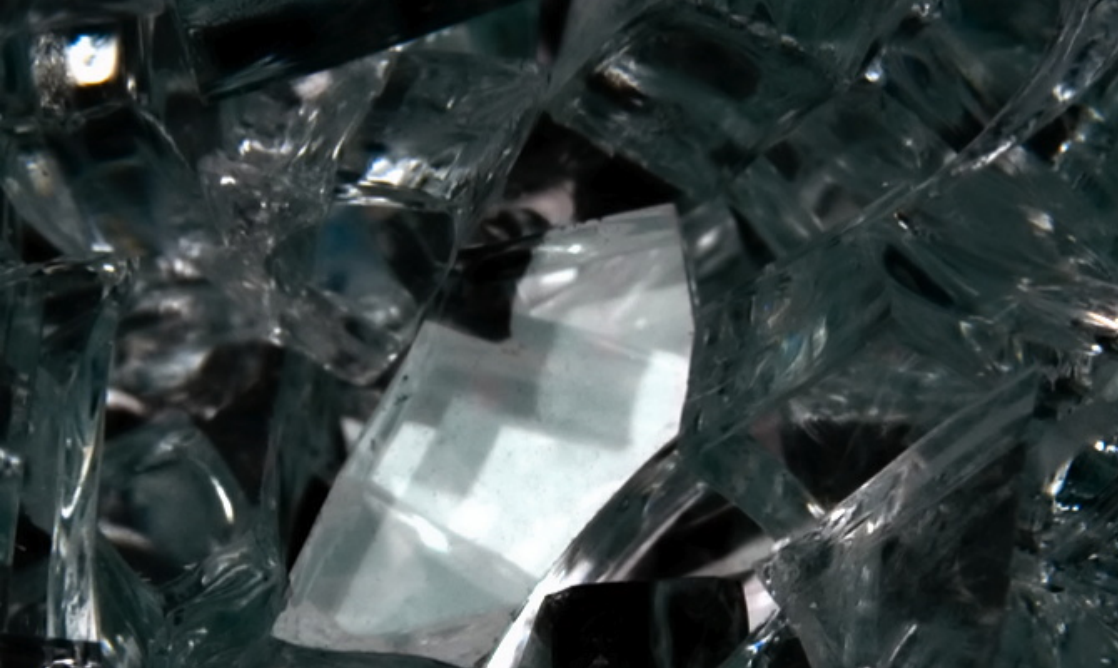

Fis
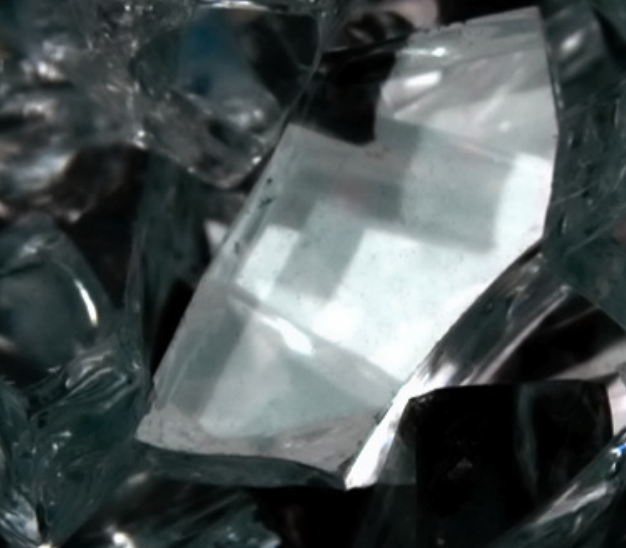


\title{
ANALYSIS OF NONSTATIONARY RANDOM PROCESSES USING SMOOTH DECOMPOSITION
}

\author{
Rubens SAmpaio AND SERgio Bellizzi
}

\begin{abstract}
Orthogonal decompositions provide a powerful tool for stochastic dynamics analysis. The most popular decomposition is the Karhunen-Loève decomposition (KLD), also called proper orthogonal decomposition. KLD is based on the eigenvectors of the correlation matrix of the random field. Recently, a modified KLD called smooth Karhunen-Loève decomposition (SD) has appeared in the literature. It is based on a generalized eigenproblem defined from the covariance matrix of the random process and the covariance matrix of the associated time-derivative random process. SD appears to be an interesting tool to extend modal analysis. Although it does not satisfy the optimality relation of KLD, and maybe is not as good a candidate for building reduced models as KLD is, SD gives access to the modal vectors independently of the mass distribution. In this paper, the main properties of SD for nonstationary random processes are explored. A discrete nonlinear system is studied through its linearization, for uncorrelated and correlated excitation, and the SD of the nonlinear system and of the linearization are compared. It seems that SD detects not only mass inhomogeneities but also nonlinearities.
\end{abstract}

\section{Introduction}

The Karhunen-Loève decomposition (KLD) method has been extensively used as a tool for analyzing random fields [Holmes et al. 1996; Lin et al. 2002; Wolter et al. 2002; Kerschen et al. 2005]. KLD reveals some coherent structures which have been advantageously used in different domains such as, for example, the stochastic finite elements method, simulation of random fields, modal analysis of nonlinear systems, and construction of reduced-order models. Depending on the discipline and the properties of the random field under study, but also on the averaging operator used to build the KLD [Bellizzi and Sampaio 2006; 2007], this decomposition has been called principal component analysis, singular value decomposition (these two in finite dimensions), and proper orthogonal decomposition. In the definition of KLD there are two inner products, one given by the normalization condition involving the standard inner product and another by the correlation; the latter, like the former, is a symmetric operator. Orthogonality is meant with respect to the normalization condition. In structural vibration, KLD has been principally applied to the displacement field, but it can be applied also to the velocity, acceleration, and displacement-velocity fields [Bellizzi and Sampaio 2009a].

Recently, a new multivariable data analysis method called smooth orthogonal decomposition (SOD) has been proposed [Chelidze and Zhou 2006]. SOD is defined from a maximization problem associated with a scalar time series of measurement but subject to a minimization constraint acting on the associated

The authors gratefully acknowledge the financial support of CNPq, Faperj, and the cooperation project 672/10 financed by CAPES and COFECUB..

Keywords: smooth decomposition, output only modal analysis, linear and nonlinear systems. 
time derivative of the time series. SOD can be used to extract normal modes and natural frequencies of multi-degree of freedom vibration systems [Zhou 2006]. Free and forced sinusoidal responses have been considered in [Chelidze and Zhou 2006] and randomly excited systems have been analyzed in [Farooq and Feeny 2008]. SOD has been formulated in term of a smooth Karhunen-Loève decomposition (SKLD) to analyze time-continuous stationary random processes in [Bellizzi and Sampaio 2009b]. The SKLD is obtained solving a generalized eigenproblem defined from the covariance matrix of the random process and the covariance matrix of its time derivative. In this paper the SKLD will be referred to as smooth decomposition (SD) since it neither has the properties of a Karhunen-Loève decomposition nor is it orthogonal in the sense of the standard inner product. There is, indeed, orthogonality with respect to inner products defined by the two correlations, displacement and velocity, as we shall see.

This work presents and discusses a nontrivial generalization of SD for time-continuous nonstationary random processes. This generalization is based on an averaging operator combining the temporal mean and mathematical expectation to build the covariance matrices of the random process and of its time derivative.

This paper is organized as follows: Section 2 extends the SD for nonstationary processes, Section 3 extends the proprieties of the SD for the nonstationary case, Section 4 gives a mechanical interpretation of the SD, Section 5 shows some numerical examples, and, finally, Section 6 presents some conclusions.

\section{Smooth decomposition}

Our goal here is to extend to nonstationary time processes the smooth decomposition introduced in [Bellizzi and Sampaio 2009b] as a smooth Karhunen-Loève decomposition for stationary random processes.

Let $\{\boldsymbol{U}(t), t \in \mathbb{R}\}$ be a $\mathbb{R}^{n}$-valued random process indexed by $\mathbb{R}$. We assume that $\{\boldsymbol{U}(t), t \in \mathbb{R}\}$ is a second-order process and admits a time-derivative process $\{\dot{\boldsymbol{U}}(t), t \in \mathbb{R}\}$ which is also a second-order process. With these assumptions, the covariance matrices of $\{\boldsymbol{U}(t), t \in \mathbb{R}\}$ and $\{\dot{\boldsymbol{U}}(t), t \in \mathbb{R}\}$, denoted $\boldsymbol{R}_{\boldsymbol{U}}(t)=\mathbb{E}\left(\boldsymbol{U}(t)^{T} \boldsymbol{U}(t)\right)$ and $\boldsymbol{R}_{\dot{\boldsymbol{U}}}(t)=\mathbb{E}\left(\dot{\boldsymbol{U}}(t)^{T} \dot{\boldsymbol{U}}(t)\right)$, respectively, are time dependent. Without loss of generality, we will also assume that $\{\boldsymbol{U}(t), t \in \mathbb{R}\}$ is a zero-mean random process and that $\boldsymbol{R}_{\boldsymbol{U}}(t)$ and $\boldsymbol{R}_{\dot{U}}(t)$ are symmetric positive definite.

In the case of stationary processes (that is, $\boldsymbol{R}_{\boldsymbol{U}}(t)$ and $\boldsymbol{R}_{\dot{U}}(t)$ do not depend on time), the smooth decomposition of $\{\boldsymbol{U}(t), t \in \mathbb{R}\}$ is defined (see [Bellizzi and Sampaio 2009b]) recursively by the maximum optimization problem

$$
\max _{\Gamma \in \mathbb{R}^{n}} \frac{\mathbb{E}\left(\langle\boldsymbol{U}(t), \Gamma\rangle^{2}\right)}{\mathbb{E}\left(\langle\dot{\boldsymbol{U}}(t), \Gamma\rangle^{2}\right)},
$$

where $\left\langle>\right.$ denotes the inner product in $\mathbb{R}^{n}$.

In the case of nonstationary processes, the objective function (see (2-1)) is time dependent and as in [Bellizzi and Sampaio 2006], where KLD has been proposed for nonstationary random processes, the time variable has to be included in the averaging operation. Let $t_{i}$ and $t_{f}$ be two positive constants with $t_{i}<t_{f}$. The ratio

$$
\frac{\frac{1}{t_{f}-t_{i}} \int_{t_{i}}^{t_{f}} \mathbb{E}\left(\langle\boldsymbol{U}(t), \Gamma\rangle^{2}\right) d t}{\frac{1}{t_{f}-t_{i}} \int_{t_{i}}^{t_{f}} \mathbb{E}\left(\langle\dot{\boldsymbol{U}}(t), \Gamma\rangle^{2}\right) d t}
$$


can be considered to define the smooth decomposition. The objective function (2-2) can be written

$$
\frac{\Gamma^{T} \mathfrak{R}_{U}^{t_{i}, t_{f}} \Gamma}{\Gamma^{T} \mathfrak{R}_{\dot{U}}^{t_{i}, t_{f}} \Gamma},
$$

where

$$
\mathfrak{R}_{U}^{t_{i}, t_{f}}=\frac{1}{t_{f}-t_{i}} \int_{t_{i}}^{t_{f}} \boldsymbol{R}_{\boldsymbol{U}}(t) d t, \quad \mathfrak{R}_{\dot{U}}^{t_{i}, t_{f}}=\frac{1}{t_{f}-t_{i}} \int_{t_{i}}^{t_{f}} \boldsymbol{R}_{\dot{U}}(t) d t
$$

showing that the quotient depends on the covariance matrices of $\left\{\boldsymbol{U}(t), t \in\left[t_{f}, t_{i}\right]\right\}$ and $\left\{\dot{\boldsymbol{U}}(t), t \in\left[t_{f}, t_{i}\right]\right\}$. The vectors that yield the extrema of

are solutions of the eigenproblem

$$
\max _{\Gamma \in \mathbb{R}^{n}} \frac{\Gamma^{T} \mathfrak{R}_{U}^{t_{i}, t_{f}} \Gamma}{\Gamma^{T} \mathfrak{R}_{\dot{U}}^{t_{i}, t_{f}} \Gamma}
$$

$$
\mathfrak{R}_{U}^{t_{i}, t_{f}} \boldsymbol{\Gamma}_{k}=\sigma_{k} \mathfrak{R}_{\dot{U}}^{t_{i}, t_{f}} \boldsymbol{\Gamma}_{k} .
$$

The SD of the random process will then be given by

$$
\boldsymbol{U}(t)=\sum_{k=1}^{n} \zeta_{k}(t) \boldsymbol{\Gamma}_{k},
$$

where the vectors $\boldsymbol{\Gamma}_{k}$ solve the generalized eigenproblem (2-6) and the scalar random processes, $\zeta_{k}(t)$, are given by

$$
\zeta_{k}(t)=\frac{\boldsymbol{\Gamma}_{k}^{T} \Re_{U}^{t_{i}, t_{f}} \boldsymbol{U}(t)}{\boldsymbol{\Gamma}_{k}^{T} \mathfrak{R}_{\boldsymbol{U}}^{t_{i}, t_{f}} \boldsymbol{\Gamma}_{k}}=\frac{\boldsymbol{\Gamma}_{k}^{T} \mathfrak{R}_{\dot{\boldsymbol{U}}}^{t_{i}, t_{f}} \boldsymbol{U}(t)}{\boldsymbol{\Gamma}_{k}^{T} \mathfrak{R}_{\dot{U}}^{t_{i}, t_{f}} \boldsymbol{\Gamma}_{k}} .
$$

Note that the scalar processes $\left\{\zeta_{k}(t)\right\}$ can be defined from either $\mathfrak{R}_{U}^{t_{i}, t_{f}}$ or $\mathfrak{R}_{U}^{t_{i}, t_{f}}$; that is, they do not depend on which of these two covariance matrices is used.

The following notation is used: the eigenvalues $\sigma_{k}$ are called the smooth values (SVs) $\left(\boldsymbol{\Sigma}=\operatorname{diag}\left(\sigma_{k}\right)\right)$, the eigenvectors $\boldsymbol{\Gamma}_{k}$ are called the smooth modes (SMs) $\left(\boldsymbol{\Gamma}=\left[\boldsymbol{\Gamma}_{1} \boldsymbol{\Gamma}_{1} \cdots \boldsymbol{\Gamma}_{n}\right]\right)$, and the scalar random processes $\left\{\zeta_{k}(t)\right\}$ are called the smooth components (SCs). All these quantities depend on the time interval $\left[t_{i}, t_{f}\right]$.

The generalized eigenproblem (2-6) is a temporal version (for nonstationary random processes) of the generalized eigenvalue problem introduced in [Bellizzi and Sampaio 2009b] to characterize the SD of the stationary process. In addition, in the definition (2-6) only the generalized covariance matrices $\mathfrak{R}_{U}^{t_{i}, t_{f}}$ and $\mathfrak{R}_{U}^{t_{i}, t_{f}}$ are used, no other operator is necessary. It is important (and trivial) to note that, if the random process is stationary, the covariance matrices reduce to the stationary ones (as described in [Bellizzi and Sampaio 2009b]) and, if the vector signal is deterministic, the covariance matrices reduce to the time-average ones (as described in [Chelidze and Zhou 2006]). We will show that the results are, of course, similar to the ones presented in [Chelidze and Zhou 2006; Farooq and Feeny 2008; Bellizzi and Sampaio 2009b], but now, since one relies on the covariance matrices, one has a powerful computation tool not available before (see, for example, [Quaranta et al. 2008]).

Finally, the quotient used to define the SD differs significantly from that used to define the classical Karhunen-Loève decomposition [Bellizzi and Sampaio 2006]. In the SD case, the denominator takes 
the covariance matrix of the time-derivative process $\{\dot{\boldsymbol{U}}(t), t \in \mathbb{R}\}$ into account which justifies the name smooth decomposition since the idea is to have a small rate-of-variation of the process.

\section{Some properties of smooth decomposition}

The classical properties as established in the case of stationary processes [Bellizzi and Sampaio 2009b] are extended to the nonstationary case.

3A. Properties of $S V$, $S M$, and $S C$. The matrices $\mathfrak{R}_{U}^{t_{i}, t_{f}}$ and $\mathfrak{R}_{U}^{t_{i}, t_{f}}$ being symmetric positive definite implies that all the SVs (eigenvalues) $v_{k}$ are strictly positive and the set of the vectors $\boldsymbol{\Gamma}_{k}$ (the SMs) constitutes a basis which is orthogonal with respect to both covariance matrices $\Re_{U}^{t_{i}, t_{f}}$ and $\mathfrak{R}_{\dot{U}}^{t_{i}, t_{f}}$. Note that the SMs are unique up to a scaling constant.

The scalar processes $\left\{\zeta_{k}(t), t \in \mathbb{R}\right\}$ are correlated:

$$
\frac{1}{t_{f}-t_{i}} \int_{t_{i}}^{t_{f}} \mathbb{E}\left(\zeta_{k}(t) \zeta_{l}(t)\right) d t=\frac{\boldsymbol{\Gamma}_{k}^{T} \Re_{U}^{t_{i}, t_{f}} \Re_{U}^{t_{i}, t_{f}} \Re_{U}^{t_{i}, t_{f}} \boldsymbol{\Gamma}_{l}}{\boldsymbol{\Gamma}_{k}^{T} \mathfrak{R}_{U}^{t_{i}, t_{f}} \boldsymbol{\Gamma}_{k} \boldsymbol{\Gamma}_{l}^{T} \mathfrak{R}_{U}^{t_{i}, t_{f}} \boldsymbol{\Gamma}_{l}}=\frac{\boldsymbol{\Gamma}_{k}^{T} \mathfrak{R}_{\dot{U}}^{t_{i}, t_{f}} \mathfrak{R}_{\dot{U}}^{t_{i}, t_{f}} \mathfrak{R}_{\dot{U}}^{t_{i}, t_{f}} \boldsymbol{\Gamma}_{l}}{\boldsymbol{\Gamma}_{k}^{T} \mathfrak{R}_{\dot{U}}^{t_{i}, t_{f}} \boldsymbol{\Gamma}_{k} \boldsymbol{\Gamma}_{l}^{T} \mathfrak{R}_{\dot{U}}^{i_{i}, t_{f}} \boldsymbol{\Gamma}_{l}} .
$$

So, the SVs are not related to energy distribution and, of course, the SD does not satisfy the standard optimality relationship as the Karhunen-Loève decomposition does. So, properly speaking, the SD is not a Karhunen-Loève decomposition. The introduction of regularity has then its drawbacks.

3B. Linear transformation of the SD. Let $\left\{\boldsymbol{V}(t), t \in\left[t_{i}, t_{f}\right]\right\}$ be a $\mathbb{R}^{n}$-valued random process defined as

$$
\boldsymbol{V}(t)=\boldsymbol{A} \boldsymbol{U}(t),
$$

where $\boldsymbol{A}$ is an invertible matrix.

From the relations $\boldsymbol{R}_{\boldsymbol{V}}(t)=\boldsymbol{A} \boldsymbol{R}_{\boldsymbol{U}}(t) \boldsymbol{A}^{T}$ and $\boldsymbol{R}_{\dot{\boldsymbol{V}}}(t)=\boldsymbol{A} \boldsymbol{R}_{\dot{\boldsymbol{U}}}(t) \boldsymbol{A}^{T}$ it can be shown that the SVs of $\left\{\boldsymbol{V}(t), t \in\left[t_{i}, t_{f}\right]\right\}$ coincide with the SVs of $\left\{\boldsymbol{U}(t), t \in\left[t_{i}, t_{f}\right]\right\}$ and the sets of the SMs satisfy

$$
\boldsymbol{\Gamma}_{k}(V)=\boldsymbol{A}^{-T} \boldsymbol{\Gamma}_{k}(U),
$$

where $\boldsymbol{\Gamma}_{k}(U)$ (respectively, $\left.\boldsymbol{\Gamma}_{k}(V)\right)$ denotes a SM of $\left\{\boldsymbol{U}(t), t \in\left[t_{i}, t_{f}\right]\right\}$ (respectively, of $\left\{\boldsymbol{V}(t), t \in\left[t_{i}, t_{f}\right]\right\}$ ). Finally, following (2-8), the SCs are invariant with respect to linear change of variables if and only if $\boldsymbol{A} \boldsymbol{A}^{T}=\boldsymbol{I}$.

\section{Mechanical interpretation of the SD}

4A. Discrete linear case. Consider a discrete mechanical system with $n$ degrees of freedom. Let $\boldsymbol{U}(t)$ be the displacement vector at instant $t$. We assume that $\boldsymbol{U}(t)$ satisfies the initial-value problem

$$
\begin{gathered}
\boldsymbol{M} \ddot{\boldsymbol{U}}(t)+\boldsymbol{C} \dot{\boldsymbol{U}}(t)+\boldsymbol{K} \boldsymbol{U}(t)=\boldsymbol{F}(t), \quad t \in\left[0, t_{f}\right], \\
\boldsymbol{U}(0)=\boldsymbol{U}_{0}, \quad \dot{\boldsymbol{U}}(0)=\dot{\boldsymbol{U}}_{0},
\end{gathered}
$$

where $\boldsymbol{M}, \boldsymbol{C}$, and $\boldsymbol{K}$ are symmetric square matrices with dimensions $n \times n$. The vectors $\boldsymbol{U}_{0}$ and $\dot{\boldsymbol{U}}_{0}$ define the initial conditions of the motion at $t=0$, and $\left\{\boldsymbol{F}(t), t \in\left[0, t_{f}\right]\right\}$ is a random vector process. Without loss of generality, we have assumed that $t_{i}=0$. 
The linear normal modes (LNM) are classically defined from the free responses of the associated undamped system as

$$
K 8=M 8 \Omega^{2},
$$

where $\mathbf{8}=\left[\mathbf{8}_{1} \cdots \mathbf{8}_{i} \cdots \mathbf{8}_{n}\right]$ denotes the modal matrix with the normalization condition $\mathbf{8}^{T} \boldsymbol{M 8}=\boldsymbol{I}$ which implies that $\mathbf{8}^{T} \boldsymbol{K} \mathbf{8}=\boldsymbol{\Omega}^{2}=\operatorname{diag}\left(\omega_{i}^{2}\right)\left(\omega_{i}^{2}\right.$ and $\mathbf{8}_{i}$ denote the squared resonance frequencies and the associated normal-mode vectors).

Here we focus on (4-1) with proportional damping. Note that in this case the matrix $\mathbf{8}^{T} \boldsymbol{C 8}$ is also diagonal. In this section the aim is to establish when and how the SMs and the SVs, defined in Section 2 (which were based on forced responses), can be used to determine the LNM. This part of the study, which is in line with the results presented in [Kerschen and Golinval 2002; Wolter et al. 2002; Feeny and Liang 2003; Chelidze and Zhou 2006], will be restricted to the case where the excitation is a white-noise random process with zero mean (that is, $\boldsymbol{R}_{F}(\tau)=\mathbb{E}\left(\boldsymbol{F}(t+\tau) \boldsymbol{F}^{T}(t)\right)=\boldsymbol{S}_{F} \delta(\tau)$, where $\boldsymbol{S}_{F}$ is a constant symmetric matrix) and the initial conditions $\left(\boldsymbol{U}_{0}\right.$ and $\left.\dot{\boldsymbol{U}}_{0}\right)$ are two random vectors with zero mean.

4A1. $S D$ and modal analysis. Introducing the modal-displacement vector $\boldsymbol{Q}(t)$ with

$$
\boldsymbol{U}(t)=\boldsymbol{\Phi} \boldsymbol{Q}(t)=\sum_{i=1}^{n} \boldsymbol{\Phi}_{i} Q_{i}(t),
$$

the equation of motion (4-1) can be equivalently replaced by

$$
\ddot{\boldsymbol{Q}}(t)+\boldsymbol{\Theta} \dot{\boldsymbol{Q}}(t)+\boldsymbol{\Omega}^{2} \boldsymbol{Q}(t)=\boldsymbol{\Phi}^{T} \boldsymbol{F}(t), \quad t \in\left[0, t_{f}\right],
$$

with $\boldsymbol{\Theta}=\boldsymbol{\Phi}^{T} \boldsymbol{C} \boldsymbol{\Phi}=\operatorname{diag}\left(2 \tau_{i} \omega_{i}\right)$.

The evolution of the covariance matrix, $\boldsymbol{R}_{\mathbb{Q}}(t)=\mathbb{E}\left(\mathbb{Q}(t) \mathbb{Q}^{T}(t)\right)$, of $\mathbb{Q}(t)=\left(\boldsymbol{Q}^{T}(t), \dot{Q}^{T}(t)\right)^{T}$ is given by (see, for example, in [Bellizzi and Sampaio 2006])

$$
\begin{aligned}
& \dot{\boldsymbol{R}}_{\mathbb{Q}}(t)=\boldsymbol{A}_{\mathbb{Q}} \boldsymbol{R}_{\mathbb{Q}}(t)+\boldsymbol{R}_{\mathbb{Q}}(t) \boldsymbol{A}_{\mathbb{Q}}^{T}+\boldsymbol{D}_{\mathbb{Q}}, \quad t \in\left[0, t_{f}\right], \\
& \boldsymbol{R}_{\mathbb{Q}}(0)=\boldsymbol{R}_{\mathbb{Q}_{0}},
\end{aligned}
$$

where

$$
\boldsymbol{A}_{\mathbb{Q}}=\left(\begin{array}{cc}
\mathbf{0} & \boldsymbol{I} \\
-\boldsymbol{\Theta} & -\boldsymbol{\Omega}^{2}
\end{array}\right), \quad \boldsymbol{D}_{\mathbb{Q}}=\left(\begin{array}{cc}
\mathbf{0} & \mathbf{0} \\
\mathbf{0} & \boldsymbol{\Phi}^{T} \boldsymbol{S}_{F} \boldsymbol{\Phi}
\end{array}\right),
$$

and $\boldsymbol{R}_{\mathbb{Q}_{0}}$ is easily deduced from $\boldsymbol{R}_{\mathbb{U}_{0}}=\mathbb{E}\left(\mathbb{U}_{0} \mathbb{U}_{0}^{T}\right)$ with $\mathbb{U}=\left(\boldsymbol{U}_{0}^{T}, \dot{\boldsymbol{U}}_{0}^{T}\right)^{T}$.

If the matrix $\boldsymbol{\Phi}^{T} \boldsymbol{S}_{F} \boldsymbol{\Phi}$ is diagonal (that is, if the modal-excitation terms $\boldsymbol{\Phi}_{i}^{T} \boldsymbol{F}(t)$ in (4-4) are uncorrelated) and if $\boldsymbol{R}_{\mathbb{Q}_{0}}$ is also diagonal then, from (4-5), it can be shown that the covariance matrix $\boldsymbol{R}_{\mathbb{Q}}(t)$ is partitioned into four blocks and each block is a $n \times n$ diagonal matrix. Hence, for all $t \in\left[0, t_{f}\right]$, the covariance matrices $\boldsymbol{R}_{\boldsymbol{Q}}(t)$ and $\boldsymbol{R}_{\dot{\boldsymbol{Q}}}(t)$ of the transient responses $\left\{\boldsymbol{Q}(t), t \in\left[0, t_{f}\right]\right\}$ and $\left\{\dot{\boldsymbol{Q}}(t), t \in\left[0, t_{f}\right]\right\}$ are diagonal. Integrating over $\left[0, t_{f}\right]$ we deduce that the generalized covariance matrices $\mathfrak{R}_{\dot{Q}}^{0, t_{f}}$ and $\mathfrak{R}_{\dot{\boldsymbol{Q}}}^{0, t_{f}}$ are also diagonal.

Solving (2-6), the SMs associated with $\left\{\boldsymbol{Q}(t), t \in\left[0, t_{f}\right]\right\}$ are equal to the vectors of the canonical basis of $\mathbb{R}^{n}$. Now using the linear relation (4-3), we can easily deduce (see (3-3)) that the SMs of $\left\{\boldsymbol{U}(t), t \in\left[0, t_{f}\right]\right\}$ are given by

$$
\boldsymbol{\Gamma}=\boldsymbol{\Phi}^{-T}, \quad \text { where } \boldsymbol{\Gamma}=\left[\boldsymbol{\Gamma}_{1} \cdots \boldsymbol{\Gamma}_{n}\right]
$$


We have here extended the result established in [Bellizzi and Sampaio 2009b] for the stationary case. Unfortunately, in the case of transient responses, it is not possible to relate the SVs $(\boldsymbol{\Sigma})$ to natural frequencies of the mechanical system as it is the case for stationary responses where, under the same assumption on $\boldsymbol{\Phi}^{T} \boldsymbol{S}_{F} \boldsymbol{\Phi}$, the following relationship holds:

$$
\boldsymbol{\Sigma}=\left(\boldsymbol{\Omega}^{2}\right)^{-1}
$$

It is interesting to note that, as indicated in [Chelidze and Zhou 2006], no assumption on the mass matrix $\boldsymbol{M}$ is needed to relate the LNMs to the SMs.

4A2. Influence of the mass inhomogeneity on the SM. An interesting property of the SMs is their sensitivity to mass inhomogeneity. Combining the two equations

$$
\Gamma=\boldsymbol{\Phi}^{-T}, \quad \boldsymbol{\Phi}^{T} \boldsymbol{M} \boldsymbol{\Phi}=\boldsymbol{I},
$$

the SM matrix reads as $\boldsymbol{\Gamma}=\boldsymbol{M \Phi}$. In the case of mass inhomogeneity - that is, when the mass matrix is diagonal with entries $m_{i}$, with not all the $m_{i}$ equal - then each SM $\boldsymbol{\Gamma}_{k}$ differs from a LNM $\boldsymbol{\Phi}_{k}$ by a scaling vector factor characterizing the mass inhomogeneity, that is,

$$
\boldsymbol{\Gamma}_{k}=\boldsymbol{V}_{\boldsymbol{M}} \cdot \boldsymbol{\Phi}_{k},
$$

where $\boldsymbol{V}_{\boldsymbol{M}}=\left(m_{1}, \ldots, m_{n}\right)^{T}$ and "." denotes the element-by-element product.

This relationship can be used in practice to localize the mass dispersion comparing the SMs and the column vectors of $\boldsymbol{\Gamma}^{-T}$. This analysis can be implemented knowing only the covariance matrices of the displacement and velocity processes.

4A3. Influence of the correlation coefficient between modal excitation terms. As we have seen above SD can be used to obtain the LNM if the modal excitation terms $\boldsymbol{\Phi}_{i}^{T} \boldsymbol{F}(t)$ are uncorrelated. In this section we will discuss the influence of the correlation coefficient between modal excitation terms considering transient responses.

Let us take a two-degree of freedom linear system of (4-1) and (4-2) with proportional damping. We assume that the matrix $\boldsymbol{\Phi}^{T} \boldsymbol{S}_{F} \boldsymbol{\Phi}$ is not diagonal and reads

$$
\boldsymbol{\Phi}^{T} \boldsymbol{S}_{F} \boldsymbol{\Phi}=\left(\begin{array}{cc}
\sigma_{11} & \rho \sqrt{\sigma_{11} \sigma_{22}} \\
\rho \sqrt{\sigma_{11} \sigma_{22}} & \sigma_{22}
\end{array}\right)
$$

where $\sigma_{11}$ and $\sigma_{22}$ denote the modal input level and $\rho$ the associated correlation coefficient.

We focus on the associated modal equation (4-4) where $\omega_{i}$ and $\tau_{i}$ (for $i=1,2$ ) denote the resonance frequencies and the associated damping ratios.

We first consider the stationary case. The covariance matrix $\hat{\boldsymbol{R}}_{\mathbb{Q}}$ of the stationary response is defined from the following Lyapunov equation:

$$
\boldsymbol{A}_{\mathbb{Q}} \hat{\boldsymbol{R}}_{\mathbb{Q}}+\hat{\boldsymbol{R}}_{\mathbb{Q}} \boldsymbol{A}_{\mathbb{Q}}^{T}+\boldsymbol{D}_{\mathbb{Q}}=0
$$


Solving (4-11) gives for $\hat{\boldsymbol{R}}_{\boldsymbol{Q}}$ and $\hat{\boldsymbol{R}}_{\dot{Q}}$ :

$$
\begin{array}{lll}
\hat{R}_{Q_{11}}=\frac{\sigma_{11}}{4 \tau_{1} \omega_{1}^{3}}, & \hat{R}_{Q_{22}}=\frac{\sigma_{22}}{4 \tau_{2} \omega_{2}^{3}}, & \hat{R}_{Q_{12}}=\rho_{Q_{12}} \sqrt{\hat{R}_{Q_{11}} \hat{R}_{Q_{22}},} \\
\hat{R}_{\dot{Q}_{11}}=\frac{\sigma_{11}}{4 \tau_{1} \omega_{1}}, & \hat{R}_{\dot{Q}_{22}}=\frac{\sigma_{22}}{4 \tau_{2} \omega_{2}}, & \hat{R}_{\dot{Q}_{12}}=\rho_{\dot{Q}_{12}} \sqrt{\hat{R}_{\dot{Q}_{11}} \hat{R}_{\dot{Q}_{22}},}
\end{array}
$$

with

$$
\rho_{Q_{12}}=\rho \frac{8 \tau_{1}^{2} r_{\omega} \sqrt{r_{\tau} r_{\omega}}\left(1+r_{\tau} r_{\omega}\right)}{\left(1-r_{\omega}^{2}\right)^{2}+4 \tau_{1}^{2}\left(1+r_{\tau} r_{\omega}\right)\left(r_{\tau}+r_{\omega}\right) r_{\omega}}, \quad \rho_{\dot{Q}_{12}}=\rho \frac{4 \sqrt{r_{\tau} r_{\omega}}}{1+r_{\tau} r_{\omega}}, \quad r_{\tau}=\frac{\tau_{2}}{\tau_{1}}, \quad r_{\omega}=\frac{\omega_{2}}{\omega_{1}} .
$$

Introducing the ratio $r_{\sigma}=\sigma_{22} / \sigma_{11}$, the stationary covariance matrices take the form

$$
\hat{\boldsymbol{R}}_{\boldsymbol{Q}}=\frac{\sigma_{11}}{4 \tau_{1} \omega_{1}^{3}}\left(\begin{array}{cc}
1 & \rho_{Q_{12}} \sqrt{\frac{r_{\sigma}}{r_{\tau} r_{\omega}^{3}}} \\
\rho_{Q_{12}} \sqrt{\frac{r_{\sigma}}{r_{\tau} r_{\omega}^{3}}} & \frac{r_{\sigma}}{r_{\tau} r_{\omega}^{3}}
\end{array}\right), \quad \hat{\boldsymbol{R}}_{\dot{\boldsymbol{Q}}}=\frac{\sigma_{11}}{4 \tau_{1} \omega_{1}}\left(\begin{array}{ccc}
1 & \rho_{\dot{Q}_{12}} \sqrt{\frac{r_{\sigma}}{r_{\tau} r_{\omega}}} \\
\rho_{\dot{Q}_{12}} \sqrt{\frac{r_{\sigma}}{r_{\tau} r_{\omega}}} & \frac{r_{\sigma}}{r_{\tau} r_{\omega}}
\end{array}\right),
$$

showing that the SVs of the stationary response defined from the generalized eigenproblem,

$$
\hat{\boldsymbol{R}}_{Q} \boldsymbol{\Gamma}_{k}=\sigma_{k} \hat{\boldsymbol{R}}_{\dot{Q}} \boldsymbol{\Gamma}_{k},
$$

depend only on the modal damping $\left(\tau_{1}, \tau_{2}\right)$, modal frequency ratio $r_{\omega}$, modal input level ratio $r_{\sigma}$, and the correlation coefficient $\rho$. Moreover, the SMs (that is, the eigenvectors $\boldsymbol{\Gamma}_{k}$ ) do not depend on the absolute values of the modal frequencies $\left(\omega_{1}, \omega_{2}\right)$.

We now consider the nonstationary case assuming zero initial conditions $(\boldsymbol{Q}(t)=0$ and $\dot{\boldsymbol{Q}}(t)=0)$. As introduced in Section 2, the SD is defined from the generalized covariance matrices

$$
\mathfrak{R}_{\boldsymbol{Q}}^{0, t_{f}}=\frac{1}{t_{f}} \int_{0}^{t_{f}} \boldsymbol{R}_{\boldsymbol{Q}}(t) d t, \quad \mathfrak{R}_{\dot{\boldsymbol{U}}}^{0, t_{f}}=\frac{1}{t_{f}} \int_{0}^{t_{f}} \boldsymbol{R}_{\dot{\boldsymbol{Q}}^{(t)}}(t)
$$

where $\boldsymbol{R}_{\boldsymbol{Q}}(t)$ and $\boldsymbol{R}_{\dot{\boldsymbol{Q}}}(t)$ solve (4-5) over [0, $\left.t_{f}\right]$. Numerical investigations are reported below for $\omega_{1}=1$, $r_{\omega}=1.5, \tau_{1}=0.01, r_{\tau}=1, \sigma_{11}=1$, and $r_{\sigma}=1$. We discuss the influence of the correlation coefficient $\rho$ between the modal excitations and the influence of $t_{f}$ on the SD. The time constant of the mechanical system is used as a time unit. The time constant is defined by $T_{c}=\max \left(1 /\left(\tau_{1} \omega_{1}\right), 1 /\left(\tau_{2} \omega_{2}\right)\right)$.

Figure 1 shows the relative errors between the canonical vectors $e_{1}=(1,0)^{T}$ and $e_{2}=(0,1)^{T}$ (the LNMs of (4-4)) and the approximations of these LNMs given by the SMs of the transient response $\left\{\boldsymbol{Q}(t), t \in\left[0, t_{f}\right]\right\}$ using (4-7), plotted versus the correlation coefficient $\rho$ and for different values of $t_{f}$ $\left(t_{f}=0.1 T_{c}, 0.2 T_{c}, 0.5 T_{c}, T_{c}\right.$, and $\left.10 T_{c}\right)$. First of all, in all the simulation results, the relative errors are small (less than 0.1) and, of course, the worse case corresponds to $\rho=1$ and $t_{f}$ small. As expected, for a given $t_{f}$, the relative error decreases as $\rho$ decreases. When $t_{f}$ increases the SD coincides with the SD given by the stationary response (see the continuous line in Figure 1).

Under the same simulation conditions, Figure 2 shows the relative errors between the resonance frequencies $\omega_{1}=1$ and $\omega_{2}=1.5$ and the approximation of these resonance frequencies given by the SMs of the transient response $\left\{\boldsymbol{Q}(t), t \in\left[0, t_{f}\right]\right\}$ using (4-8). Here also the relative errors are very small (less than 0.001). Moreover, for fixed $t_{f}$, the relative error does not depend on $\rho$ and decreases when $t_{f}$ increases. 

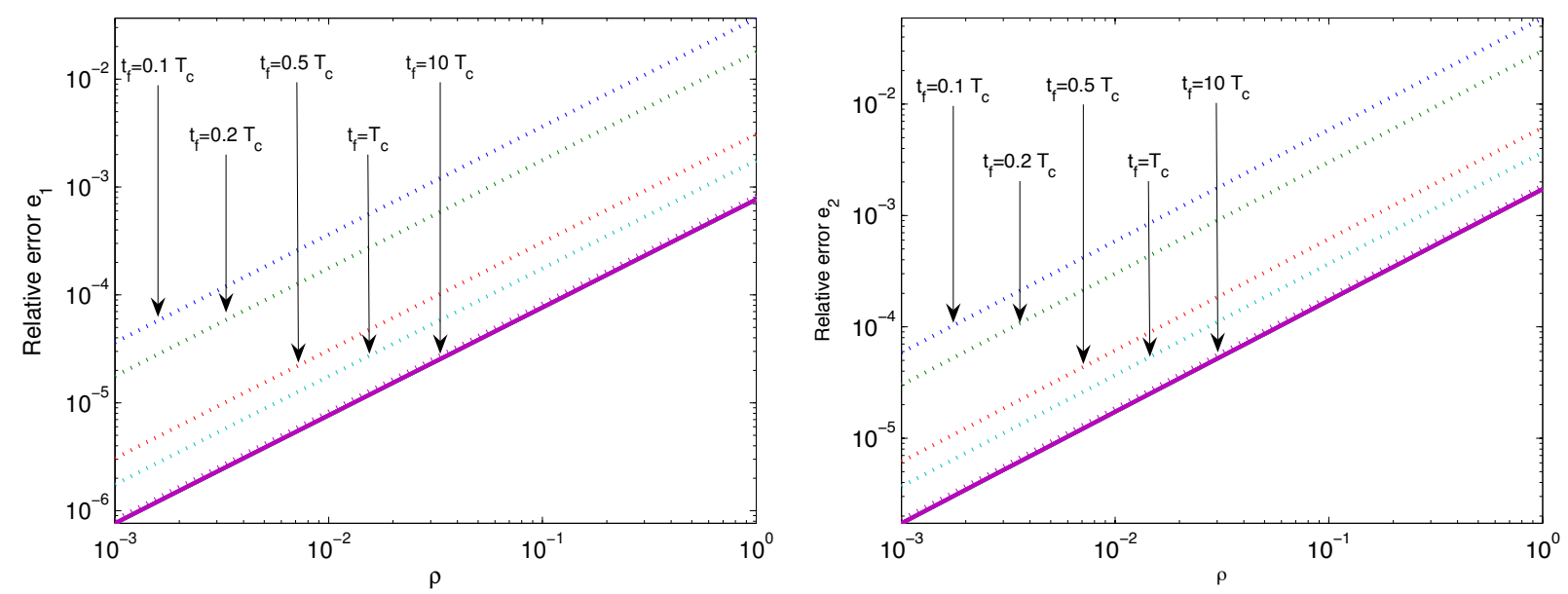

Figure 1. Relative error (using Euclidean norm vector) between the canonical vector $\left(e_{1}=(1,0)^{T}\right.$ (left) and $e_{2}=(0,1)^{T}$ (right)) and the approximation of these LNMs given by the SMs of the transient response $\left\{\boldsymbol{Q}(t), t \in\left[0, t_{f}\right]\right\}$ using (4-7) (dotted lines) and the approximation of these LNMs given by the SMs of the stationary response (continuous line).
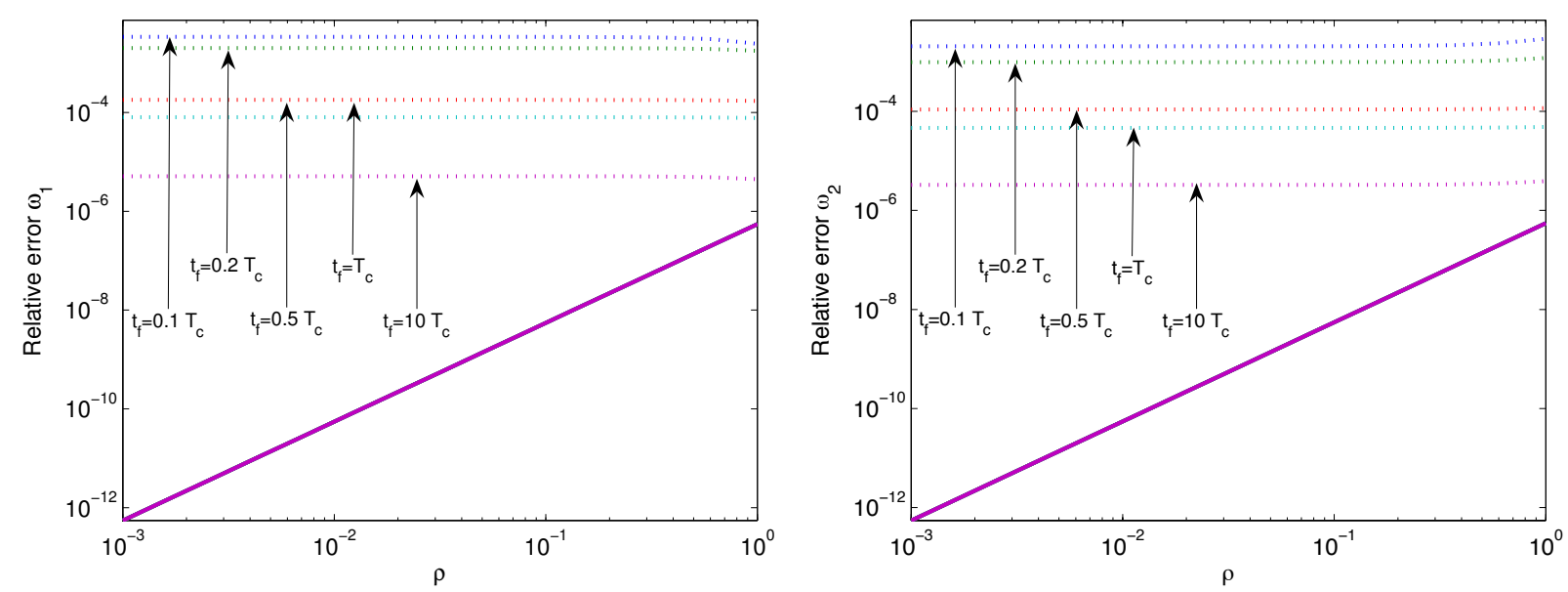

Figure 2. Relative error between the natural resonance-frequency vector $\left(\omega_{1}\right.$ (left) and $\omega_{2}$ (right)) and the approximation of these LNMs given by the SMs of the transient response $\left\{\boldsymbol{Q}(t), t \in\left[0, t_{f}\right]\right\}$ using (4-8) (dotted lines) and the approximation of these LNMs given by the SMs of the stationary response (continuous line).

4B. Discrete nonlinear case. Consider a discrete mechanical system with $n$ degrees of freedom. Let $\boldsymbol{U}(t)$ be the displacement vector at the instant $t$. We assume that $\boldsymbol{U}(t)$ satisfies the initial-value problem

$$
\begin{gathered}
\boldsymbol{M} \ddot{\boldsymbol{U}}(t)+\boldsymbol{H}(\dot{\boldsymbol{U}}(t), \boldsymbol{U}(t))=\boldsymbol{F}(t), \quad t \in\left[0, t_{f}\right] \\
\boldsymbol{U}(0)=\boldsymbol{U}_{0}, \quad \dot{\boldsymbol{U}}(0)=\dot{\boldsymbol{U}}_{0},
\end{gathered}
$$


where $\boldsymbol{M}$ is a symmetric square matrix with dimension $n \times n$ and $H$ is a $n$-vector smooth nonlinear function. The vectors $\boldsymbol{U}_{0}$ and $\dot{\boldsymbol{U}}_{0}$ define the initial conditions of the motion at $t=0$, and $\left\{\boldsymbol{F}(t), t \in\left[0, t_{f}\right]\right\}$ is a random vector process.

One rather interesting result was the difference between the SM obtained using the SD of the stationary response of the nonlinear system and the SM obtained using the SD of the stationary response of the equivalent linear system obtained using statistical linearization, as described in [Kozin 1988].

Rewriting (4-17) as a nonlinear first-order differential equation (for $\left.\mathbb{U}(t)=\left(\boldsymbol{U}(t)^{T}, \dot{\boldsymbol{U}}(t)^{T}\right)^{T}\right)$

$$
\dot{U}(t)=N(\mathbb{U}(t))+\boldsymbol{G}(t),
$$

with external random excitation $\boldsymbol{G}(t)=\left(0,\left(\boldsymbol{M}^{-1} \boldsymbol{F}(t)\right)^{T}\right)^{T}$. A suitable equivalent linear system can be written as

$$
\dot{U}(t)=\boldsymbol{L}_{\mathrm{eq}} \mathbb{U}(t)+\boldsymbol{G}(t),
$$

where the constant matrix $\boldsymbol{L}_{\text {eq }}$ is determined by

$$
\min _{\boldsymbol{L}} E\left(\|\boldsymbol{N}(\mathbb{U}(t))-\boldsymbol{L} \mathbb{U}(t)\|^{2}\right) .
$$

Under the assumption that the nonlinear system (4-19) admits a stationary ergodic probability measure, it can be shown [Kozin 1988] that the stationary covariance matrix of the nonlinear response (4-19) coincides with the stationary covariance matrix of the equivalent linear response (4-20). Hence, the SD analysis of the stationary response of the nonlinear system (4-17) gives the same results as the SD analysis of the stationary response of the equivalent linear system except for the SCs. Following the modal analysis described in the previous sections, the SD can also be viewed as a tool for modal analysis of the nonlinear system, the SMs and SVs of the nonlinear system being interpreted as in reference to the modal characteristics of the linearized system.

\section{Numerical example}

We consider a finite chain of $n$ mass points with the first one linked by a linear spring to a fixed point, the others consecutively linked one to the other, and the last one linked only to the previous mass. All the stiffness coefficients of the strings are equal and their common value is 1 . The mass values are denoted by $m_{i}\left(m_{i}>0\right)$. The system can also include isolated nonlinearities between consecutive masses of the form $\lambda_{i}\left(U_{i}(t)-U_{i-1}(t)\right)^{3}$ for $i=2, \ldots, n$. The associated equations of motion are of the form of (4-17), with

$$
\boldsymbol{M}=\left(\begin{array}{cccccc}
m_{1} & 0 & 0 & \cdots & 0 & 0 \\
0 & m_{2} & 0 & & 0 & 0 \\
0 & 0 & m_{3} & & 0 & 0 \\
\vdots & & & & & \vdots \\
0 & 0 & 0 & & m_{n-1} & 0 \\
0 & 0 & 0 & \cdots & 0 & m_{n}
\end{array}\right), \quad \boldsymbol{K}=\left(\begin{array}{rrrrrr}
2 & -1 & 0 & \cdots & 0 & 0 \\
-1 & 2 & -1 & & 0 & 0 \\
0 & -1 & 2 & & 0 & 0 \\
\vdots & & & & & \vdots \\
0 & 0 & 0 & & 2 & -1 \\
0 & 0 & 0 & \cdots & -1 & 1
\end{array}\right) .
$$

$\boldsymbol{H}$, which only depends on $\boldsymbol{U}(t)$, is easily deduced from the form of the nonlinearity. The damping matrix is chosen to be $\boldsymbol{C}=2 \tau_{1} \omega_{1} \boldsymbol{M}$, with $\tau_{1}>0$, which assures that the damping is proportional and 
fixes the damping ratio of the first linear mode. Note that the linear version of this system has been discussed in [Farooq and Feeny 2008].

Two excitation conditions will be considered:

- Uncorrelated excitation: the system is excited by a standard vector-valued white-noise process with matrix intensity

$$
\boldsymbol{S}_{F}=S_{0} \boldsymbol{M},
$$

with $S_{0}>0$. This choice ensures that, for all mass values $m_{i}, \boldsymbol{\Phi}^{T} \boldsymbol{S}_{F} \boldsymbol{\Phi}=S_{0} \boldsymbol{I}$ is always diagonal.

- Correlated excitation: the system is excited by a white-noise scalar process applied to the mass numbered $i_{\text {excit }}$, that is,

$$
\boldsymbol{F}(t)=(0 \cdots 010 \cdots 0)^{T} f(t)=\boldsymbol{P} f(t),
$$

with $\{f(t), t \in \mathbb{R}\}$ being a white-noise process with intensity $S_{0}>0$. The intensity matrix of $\{\boldsymbol{F}(t), t \in \mathbb{R}\}$ is given by $\boldsymbol{S}_{F}=S_{0} \boldsymbol{P} \boldsymbol{P}^{T}$ and hence $\boldsymbol{\Phi}^{T} \boldsymbol{S}_{F} \boldsymbol{\Phi}=S_{0}\left(\Phi_{i i_{\text {excit }}} \Phi_{j i}\right)$ is not a diagonal matrix.

The displacement and velocity histories were obtained from excitation histories by solving (4-17) over $\left[0, t_{f}\right]$ numerically using the Newmark method. The excitation histories were simulated using the procedure described in [Poirion and Soize 1989]. The following values of parameters were used: $n=10$, $m_{2}=2$, and $m_{i}=1$ for $i \neq 2, \tau_{1}=0.05, \lambda_{5}=10$, and $\lambda_{i}=0$ for $i \neq 5, S_{0}=1$, and for the correlated case, $i_{\text {excit }}=1$. Zero initial displacement and velocity were assumed. The time-discretization parameter value was chosen equal to $\Delta t=0.1$ (that is, $f_{e}=10$ ) and 65536 instants were simulated. The last-half points of the displacement and velocity histories were used to approximate the covariance matrices $\boldsymbol{R}_{\boldsymbol{U}}$ and $\boldsymbol{R}_{\dot{U}}$ of the stationary response. The simulated data were also used to estimate $\boldsymbol{L}_{\text {eq }}$ solving (4-21) and the SDs of the stationary response of the equivalent linear system (4-20) were computed solving the associated equation (4-11).

The estimated values of the resonance frequencies obtained from SD analysis are reported in Table 1. Note that the expression "resonance frequency" is a misnomer because the system is nonlinear; we will

\begin{tabular}{|lcrccc|}
\hline & Underlying & \multicolumn{3}{c|}{ Uncorrelated case } & \multicolumn{2}{c|}{ Correlated case } \\
& linear system & SD from: $(4-17)$ & $(4-20)$ & $(4-17)$ & $(4-20)$ \\
$\omega_{1}$ & 0.0236 & 0.0246 & 0.0246 & 0.0243 & 0.0243 \\
$\omega_{2}$ & 0.0665 & 0.0705 & 0.0706 & 0.0696 & 0.0696 \\
$\omega_{3}$ & 0.1068 & 0.1077 & 0.1074 & 0.1076 & 0.1073 \\
$\omega_{4}$ & 0.1515 & 0.1656 & 0.1655 & 0.1626 & 0.1626 \\
$\omega_{5}$ & 0.1960 & 0.1984 & 0.1983 & 0.1977 & 0.1978 \\
$\omega_{6}$ & 0.2327 & 0.2430 & 0.2429 & 0.2418 & 0.2417 \\
$\omega_{7}$ & 0.2491 & 0.2519 & 0.2521 & 0.2513 & 0.2514 \\
$\omega_{8}$ & 0.2740 & 0.2862 & 0.2866 & 0.2850 & 0.2850 \\
$\omega_{9}$ & 0.2980 & 0.3010 & 0.3013 & 0.2996 & 0.2997 \\
$\omega_{10}$ & 0.3132 & 1.0234 & 1.0240 & 0.4533 & 0.4533 \\
\hline
\end{tabular}

Table 1. Estimated values of the resonance frequencies obtained from SD analysis. 
use it in reference to the associated linearized system. As expected in the uncorrelated case, the estimates obtained from the nonlinear system coincide with those given by the linearized system. These values are very close to the resonance-frequency values of the underlying linear system (that is, with $\lambda_{5}=0$ ) except for the last value which is larger due to the nonlinear term. The same comments hold also for the correlated case showing that the SD properties are robust to the loss of the noncorrelation assumption. Here also the numerical value of $\omega_{10}$ is large, resulting in the effect of the nonlinear term which is however smaller than the uncorrelated case.

For the uncorrelated case, the SMs obtained from the stationary responses of the nonlinear system and the linearized system are plotted in Figure 3. As expected in the uncorrelated case (see Section 4A1), the SMs obtained from the nonlinear system coincide with those given by the linearized system. The SMs differ significantly from the normal modes of the underlying linear system, also plotted in the figure. For the first modes, the difference occurs only around the mass number 2 where the mass inhomogeneity is
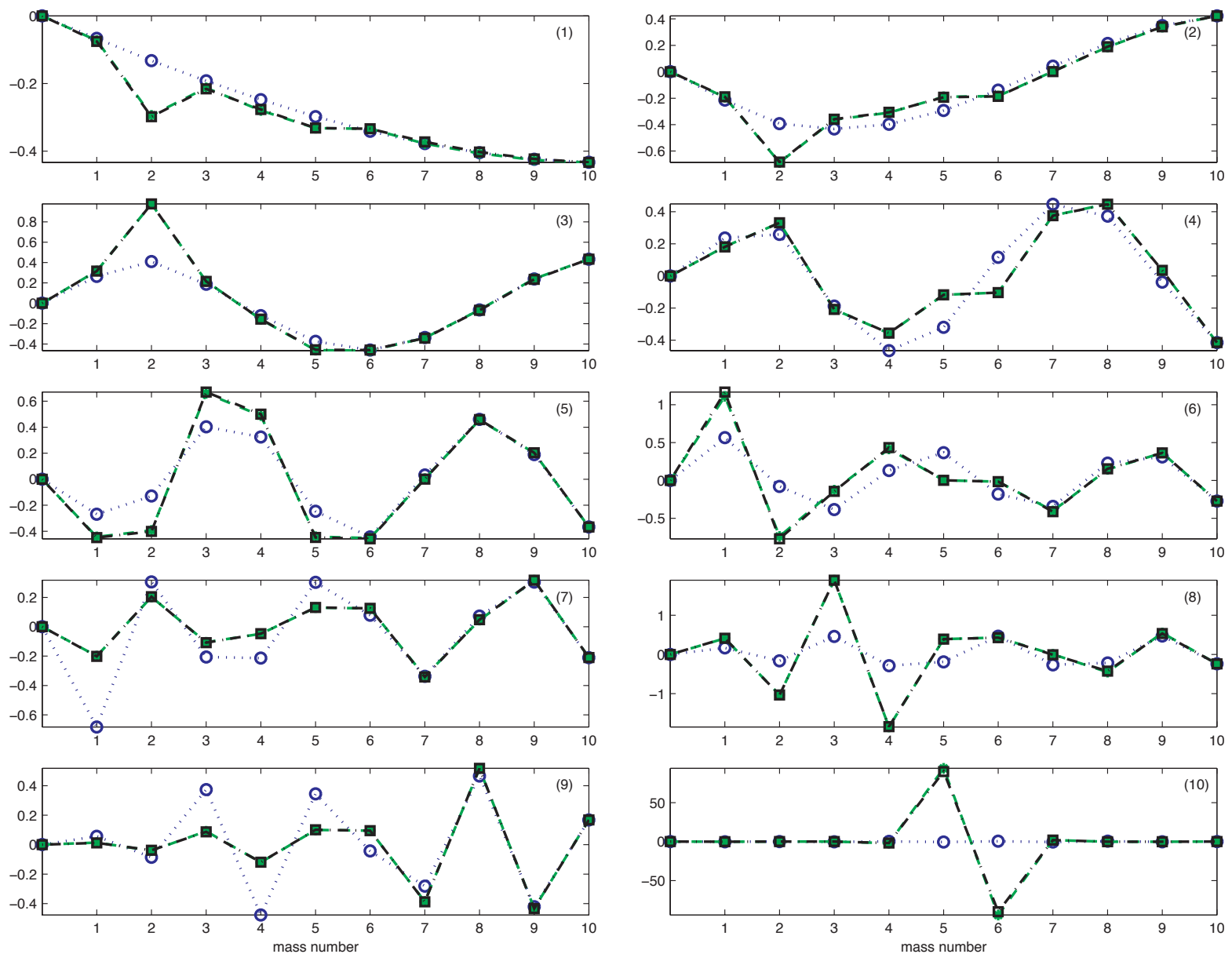

Figure 3. Uncorrelated case: the SMs (dashed lines and point markers) and the associated normal modes given by $\boldsymbol{\Gamma}^{-T}$ (dotted-dashed lines and square markers), see (4-7), obtained from the nonlinear system. The normal modes of the underlying linear system are also reported (dotted lines and circle markers). 
present. The relationship between the normal modes and the SMs is in line with (4-9). For the last mode (corresponding to $\omega_{10}$ ), the difference between the normal mode and the SM occurs around the mass numbers 5 and 6, where there is local nonlinearity. At these two masses the amplitudes of the SMs are very large and have opposite signs. The SMs, then, seem to be also sensitive to the nonlinearity of the system. In Figure 4, the modes obtained from the SMs of the stationary responses of the nonlinear system and the linearized system are plotted and compared to the normal modes of the underlying linear system. The first modes are very similar. The difference increases for the middle and higher modes. For the correlated case, the SMs obtained from the stationary responses of the nonlinear system and the linearized system are plotted in Figure 5 and compared to the normal modes of the underlying linear system. For the middle modes (vector 4 to vector 8), the nonlinear SMs differ from the linearized SMs. Here also, the SMs differ from the normal modes of the underlying linear system where the mass inhomogeneity is present (mass number 2) and where the local nonlinear term acts (mass numbers 5 and 6 ).
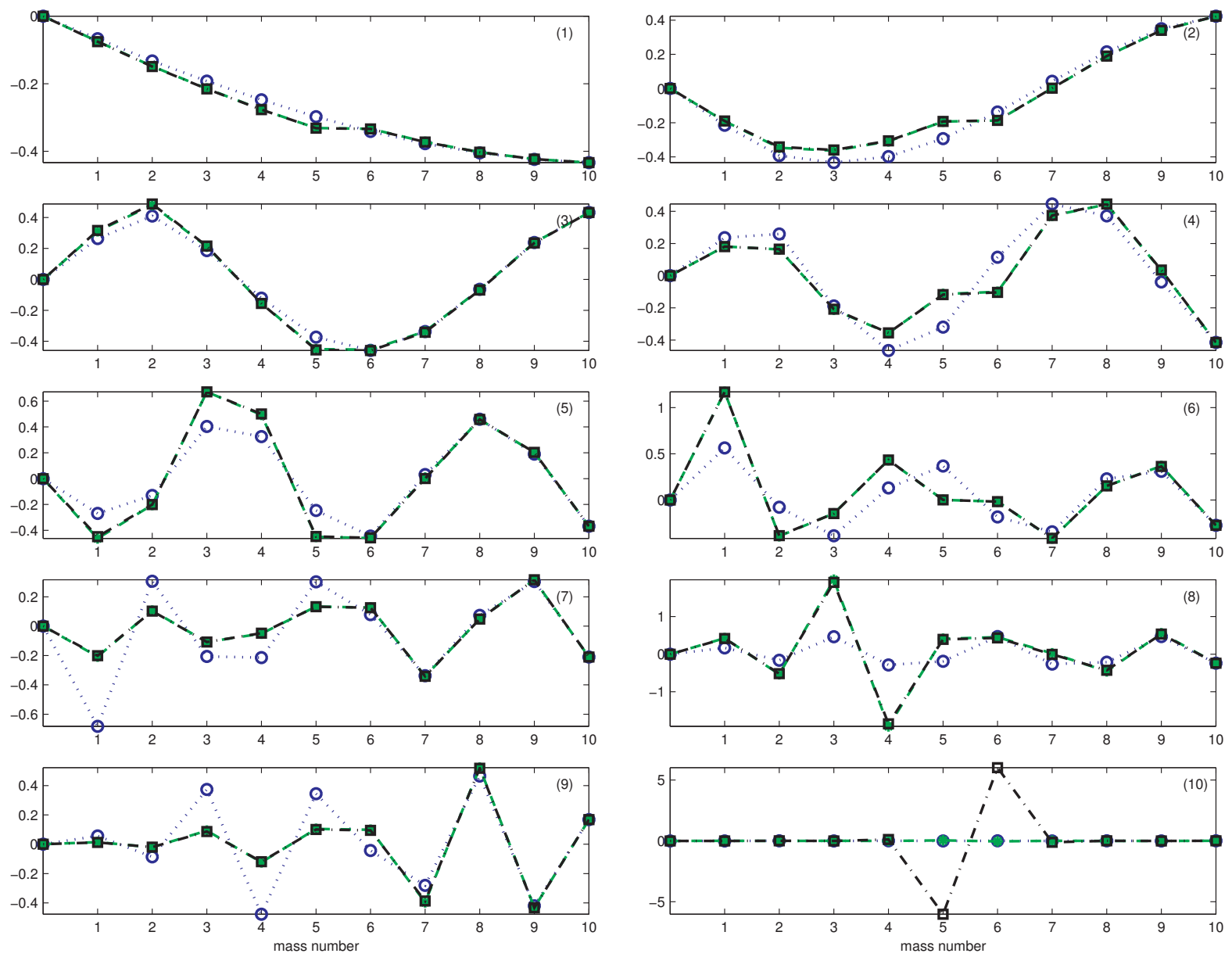

Figure 4. Uncorrelated case: the normal modes obtained from the nonlinear system (dashed lines and point markers), $\Gamma^{-T}$ (see (4-7)), and form the linearized system (dotted-dashed lines and square markers). The normal modes of the underlying linear system are also reported (dotted lines and circle markers). 

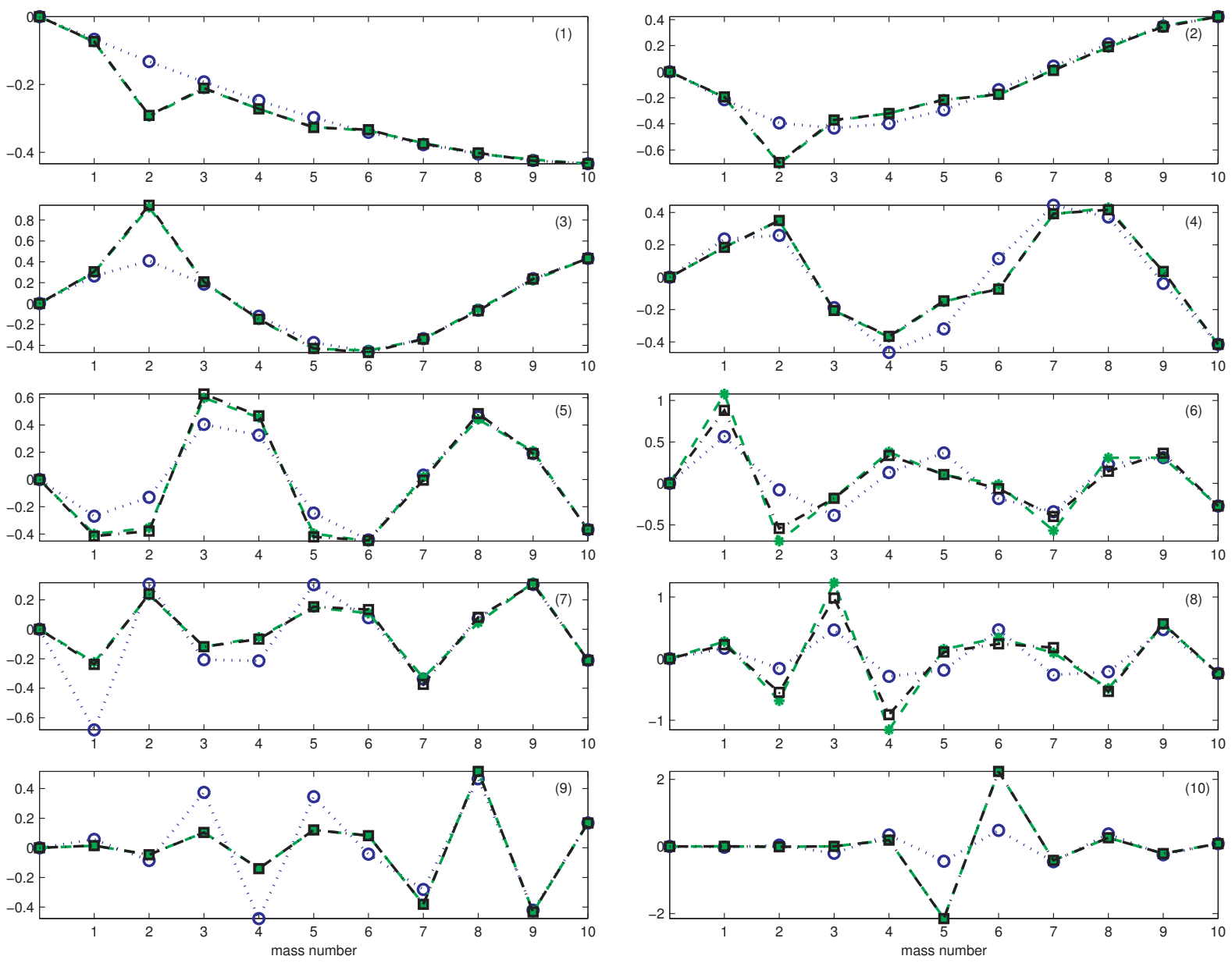

Figure 5. Correlated case: the SMs (dashed lines and point markers) and the associated normal modes given by $\Gamma^{-T}$ (dotted-dashed lines and square markers), see (4-7), obtained from the nonlinear system. The normal modes of the underlying linear system are also reported (dotted lines and circle markers).

In Figure 6, the SMs of the stationary responses of the nonlinear system are compared to the modes obtained from the SMs of the stationary responses of the nonlinear system. Here also, the SMs differ from the approximate normal modes where the mass inhomogeneity is present (mass number 2) and where the local nonlinear term acts (mass numbers 5 and 6). Note that these modes have been obtained only from data. This shows the ability of SD analysis to extract frequency, mode, and mass information.

\section{Conclusions}

In this paper, the smooth orthogonal decomposition method introduced in [Chelidze and Zhou 2006] was formulated in terms of a smooth decomposition (SD) (also called smooth Karhunen-Loève decomposition in [Bellizzi and Sampaio 2009b]) to analyze time-continuous nonstationary random processes. The SD is obtained by solving a generalized eigenproblem defined by combining the covariance matrix 

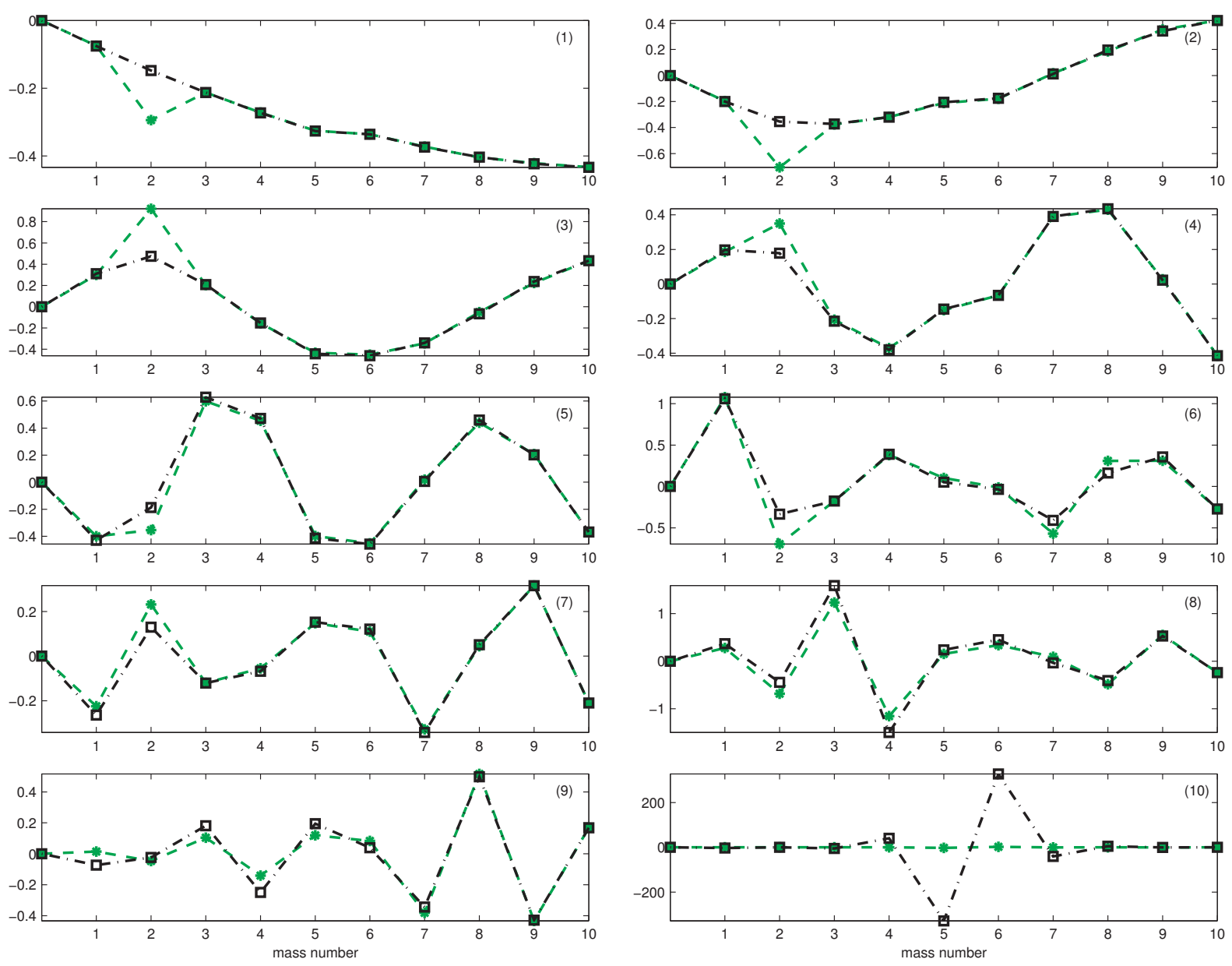

Figure 6. Correlated case: the SMs obtained from the nonlinear system (dashed lines and point markers) and the associated normal modes given by $\boldsymbol{\Gamma}^{-T}$ (dotted-dashed lines and square markers), see (4-7).

of the random field with that of the associated time-derivative random field, a constraint in the rate of variation of the field that originates the term smooth. The covariance operator is defined from an averaging operator combining the temporal mean and mathematical expectation which reduces to the mathematical expectation in the case of time-stationary random processes. Note that in this case, the definition has several computational advantages, for example, the use of Lyapunov equations to compute the covariances (see [Bellizzi and Sampaio 2006]). SD does not have the best-decomposition properties of the Karhunen-Loève decomposition (KLD) and its orthogonality is with respect to the two covariance matrices used in its definition. In the context of output-only modal analysis (that is, without excitation data), SD has several advantages with respect to KLD. If the modal forcing components are not correlated and if the damping is proportional it is possible to estimate, without condition on the mass distribution, normal modes and also, in the stationary case, resonance frequencies directly. This is true independently of the damping level. Note that if the modal forcing components are correlated, the efficiency of the SD 
method to estimate resonance frequencies and normal modes rapidly decreases when the damping level increases. Beyond the modal analysis, another interesting property of the SD is that it is possible to extract the mass distribution from the SD comparing the smooth modes (SMs) and the normal modes estimated also from the SMs. Finally, the SD can also be viewed as a tool for modal analysis of nonlinear systems. In the stationary case, the SMs and smooth values (SVs) of the nonlinear response coincide with the SMs and SVs of the response of the equivalent linear system obtained by a statistical linearization approach. Moreover under a constant mass matrix assumption, the SMs give access to the mass distribution.

\section{References}

[Bellizzi and Sampaio 2006] S. Bellizzi and R. Sampaio, "POMs analysis of randomly vibrating systems obtained from Karhunen-Loève expansion”, J. Sound Vib. 297:3-5 (2006), 774-793.

[Bellizzi and Sampaio 2007] S. Bellizzi and R. Sampaio, "Analysis of randomly vibrating systems using Karhunen-Loève expansion", pp. 1387-1395 in Proceedings of the ASME International Design Engineering Technical Conferences \& Computers and Information in Engineering Conference (Las Vegas, NV, 2007), vol. 1, ASME, New York, 2007. Paper \#DETC200734431.

[Bellizzi and Sampaio 2009a] S. Bellizzi and R. Sampaio, "Karhunen-Loève modes obtained from displacement and velocity fields: assessments and comparisons", Mech. Syst. Signal Process. 23:4 (2009), 1218-1222.

[Bellizzi and Sampaio 2009b] S. Bellizzi and R. Sampaio, "Smooth Karhunen-Loève decomposition to analyze randomly vibrating systems", J. Sound Vib. 325:3 (2009), 491-498.

[Chelidze and Zhou 2006] D. Chelidze and W. Zhou, "Smooth orthogonal decomposition-based vibration mode identification", J. Sound Vib. 292:3-5 (2006), 461-473.

[Farooq and Feeny 2008] U. Farooq and B. F. Feeny, "Smooth orthogonal decomposition for modal analysis of randomly excited systems", J. Sound Vib. 316:1-5 (2008), 137-146.

[Feeny and Liang 2003] B. F. Feeny and Y. Liang, "Interpreting proper orthogonal modes of randomly excited vibration systems", J. Sound Vib. 265:5 (2003), 953-966.

[Holmes et al. 1996] P. Holmes, J. L. Lumley, and G. Berkooz, Turbulence, coherent structures, dynamical systems and symmetry, Cambridge University Press, Cambridge, 1996.

[Kerschen and Golinval 2002] G. Kerschen and J.-C. Golinval, "Physical interpretation of the proper orthogonal modes using the singular value decomposition", J. Sound Vib. 249:5 (2002), 849-865.

[Kerschen et al. 2005] G. Kerschen, J.-C. Golinval, A. F. Vakakis, and L. A. Bergman, "The method of proper orthogonal decomposition for dynamical characterization and order reduction of mechanical systems: an overview", Nonlinear Dyn. 41:1-3 (2005), 147-169.

[Kozin 1988] F. Kozin, “The method of statistical linearization for nonlinear stochastic vibrations”, pp. 45-56 in Nonlinear stochastic dynamic engineering systems (IUTAM Symposium) (Innsbruck, 1987), edited by F. Ziegler and G. I. Schuëller, Springer, Berlin, 1988.

[Lin et al. 2002] W. Z. Lin, K. H. Lee, P. Lu, S. P. Lim, and Y. C. Liang, "The relationship between eigenfunctions of KarhunenLoève decomposition and the modes of distributed parameter vibration system”, J. Sound Vib. 256:4 (2002), 791-799.

[Poirion and Soize 1989] F. Poirion and C. Soize, "Simulation numérique des champs stochastiques Gaussiens homogènes et non homogènes", Rech. Aérosp. 1 (1989), 41-61.

[Quaranta et al. 2008] G. Quaranta, P. Masarati, and P. Mantegazza, "Continuous-time covariance approaches for modal analysis", J. Sound Vib. 310:1-2 (2008), 287-312.

[Wolter et al. 2002] C. Wolter, M. A. Trindade, and R. Sampaio, "Obtaining mode shapes through the Karhunen-Loève expansion for distributed-parameter linear systems", Shock Vib. 9:4-5 (2002), 177-192.

[Zhou 2006] W. Zhou, Multivariate analysis in vibration modal parameter identification, Ph.D. thesis, University of Rhode Island, 2006, Available at http://gradworks.umi.com/32/48/3248248.html. 
Received 20 May 2010. Revised 27 Sep 2010. Accepted 14 Nov 2010.

RUBENS SAMPAIO: rsampaio@puc-rio.br

Departamento de Engenharia Mecânica, PUC-Rio, Rua Marquês de São Vicente, 225, 22453-900 Rio de Janeiro-RJ, Brazil

SERGIO BELLIZZI: bellizzi@lma.cnrs-mrs.fr

Laboratoire de Mécanique et d'Acoustique - CNRS, 31 chemin Joseph Aiguier, 13402 Marseille, France 


\title{
JOURNAL OF MECHANICS OF MATERIALS AND STRUCTURES
}

\author{
jomms.org
}

Founded by Charles R. Steele and Marie-Louise Steele

EDITORS

Charles R. SteEle

DAVIDE BIGONI

Stanford University, USA

YASUHIDE SHINDO

University of Illinois at Urbana-Champaign, USA

Tohoku University, Japan

\section{EDITORIAL BOARD}

$\begin{aligned} \text { H. D. BUI } & \text { École Polytechnique, France } \\ \text { J. P. CARTER } & \text { University of Sydney, Australia } \\ \text { R. M. CHRISTENSEN } & \text { Stanford University, USA } \\ \text { G. M. L. GLADWELL } & \text { University of Waterloo, Canada } \\ \text { D. H. HODGES } & \text { Georgia Institute of Technology, USA } \\ \text { J. HUTCHINSON } & \text { Harvard University, USA } \\ \text { C. HWU } & \text { National Cheng Kung University, Taiwan } \\ \text { B. L. KARIHALOO } & \text { University of Wales, UK } \\ \text { Y. Y. KIM } & \text { Seoul National University, Republic of Korea } \\ \text { Z. MROZ } & \text { Academy of Science, Poland } \\ \text { D. PAMPLONA } & \text { Universidade Católica do Rio de Janeiro, Brazil } \\ \text { M. B. RUBIN } & \text { Technion, Haifa, Israel } \\ \text { A. N. SHUPIKOV } & \text { Ukrainian Academy of Sciences, Ukraine } \\ \text { T. TARNAI } & \text { University Budapest, Hungary } \\ \text { F. Y. M. WAN } & \text { University of California, Irvine, USA } \\ \text { P. WRIGGERS } & \text { Universität Hannover, Germany } \\ \text { W. YANG } & \text { Tsinghua University, China } \\ \text { F. ZIEGLER } & \text { Technische Universität Wien, Austria } \\ & \\ \text { PRODUCTION } & \text { contact@ msp.org } \\ \text { SILVIO LEVY } & \text { Scientific Editor }\end{aligned}$

Cover design: Alex Scorpan

Cover photo: Mando Gomez, www.mandolux.com

See http://jomms.org for submission guidelines.

JoMMS (ISSN 1559-3959) is published in 10 issues a year. The subscription price for 2011 is US \$520/year for the electronic version, and \$690/year (+\$60 shipping outside the US) for print and electronic. Subscriptions, requests for back issues, and changes of address should be sent to Mathematical Sciences Publishers, Department of Mathematics, University of California, Berkeley, CA 94720-3840.

JoMMS peer-review and production is managed by EditFLow ${ }^{\circledR}$ from Mathematical Sciences Publishers.

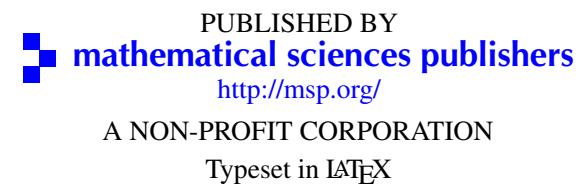

Copyright (C2011 by Mathematical Sciences Publishers 


\section{Journal of Mechanics of Materials and Structures}

\section{Volume 6, No. 7-8}

September-October 2011

\section{Special issue \\ Eleventh Pan-American Congress \\ of Applied Mechanics (PACAM XI)}

Preface

Adair R. Aguiar

949

Influence of specimen geometry on the Portevin-Le Châtelier effect due to dynamic strain aging

for the AA5083-H116 aluminum alloy

Rodrigo Nogueira de Codes and Ahmed Benallal

Dispersion relations for SH waves on a magnetoelectroelastic heterostructure with imperfect

interfaces

J. A. Otero, H. Calas, R. Rodríguez, J. Bravo, A. R. Aguiar and G. Monsivais

Numerical linear stability analysis of a thermocapillary-driven liquid bridge with magnetic stabilization

Yue Huang and Brent C. Houchens

Numerical investigation of director orientation and flow of nematic liquid crystals in a planar 1:4 expansion Pedro a. Cruz, Murilo F. Tomé, IAin W. Stewart and Sean McKee

Critical threshold and underlying dynamical phenomena in pedestrian-induced lateral vibrations of footbridges

Stefano LenCI and LAURA MARCHEgGiani

Free vibration of a simulation CANDU nuclear fuel bundle structure inside a tube

XUAN ZHANG and SHUdONG Yu

Nonlinear dynamics and sensitivity to imperfections in Augusti's model

D. Orlando, P. B. Gonçalves, G. Rega and S. LenCi

Active control of vortex-induced vibrations in offshore catenary risers: A nonlinear normal mode approach

CArlos E. N. MAZzilli and César T. SANCheS

Nonlinear electromechanical fields and localized polarization switching of piezoelectric macrofiber composites

Yasuhide Shindo, Fumio Narita, KoJi SATo and Tomo TAKeda

1089

Three-dimensional BEM analysis to assess delamination cracks between two transversely isotropic materials

Nicolás O. Larrosa, Jhonny E. Ortiz and Adrián P. Cisillino

Porcine dermis in uniaxial cyclic loading: Sample preparation, experimental results and modeling

A. E. Ehret, M. Hollenstein, E. MAzzA and M. Itskov

Analysis of nonstationary random processes using smooth decomposition

Rubens SAMpaio and Sergio Bellizzi

Perturbation stochastic finite element-based homogenization of polycrystalline materials

S. LePage, F. V. Stump, I. H. Kim and P. H. Geubelle

A collocation approach for spatial discretization of stochastic peridynamic modeling of fracture

Georgios I. Evangelatos and POL D. SPANOS 